\title{
Third-order nonlinear optics in KTP ridge waveguides
}

\author{
Augustin Vernay ${ }^{1}$, Véronique Boutou ${ }^{1}$, Corinne Félix ${ }^{1}$, David Jegouso ${ }^{1}$, Florent Bassignot ${ }^{2}$, Mathieu \\ Chauvet $^{3}$, Benoit Boulanger ${ }^{1}$ \\ 1. Univ. Grenoble Alpes, CNRS, Institut Néel, 38000 Grenoble, France \\ 2. Femto-Engineering, 15B avenue des Montboucons, 25000 Besançon, France \\ 3. FEMTO-ST Institute, UMR CNRS 6174, Université de Franche-Comté, 15B avenue des Montboucons, 25000 Besançon, France
}

Ridges waveguides carved in bulk nonlinear crystals such as $\mathrm{LiNbO}_{3}$ [1] or $\mathrm{KTiOPO}_{4}(\mathrm{KTP})$ [2] are a good alternative to reach a strong confinement of electromagnetic waves with large second-order or third-order nonlinearity. We report here on our most recent experiments where we show the possibility to shape the birefringence phase-matching (BPM) conditions for direct Third-Harmonic Generation (THG: $\omega+\omega+\omega \rightarrow 3 \omega$ ) in micrometric KTP ridge waveguides by acting on their transverse dimensions. This study is the preliminary step for designing quantum optical experiments based on Triple Photons Generation (TPG: $3 \omega \rightarrow \omega+\omega+\omega)$ that is the reverse process of THG, thus exhibiting the same BPM conditions.

A first step in our study has been to model the wavelength dispersion of the effective refractive indices as a function of the transverse dimension $d$ of a squared-transverse-section ridge. Then it has been possible to calculate the THG BPM conditions as a function of $d$. Using these data, we chose to design a guide with $d=6.2 \mu \mathrm{m}$ in order to reach phase-matching at a fundamental wavelength $\lambda_{\omega}^{B P M}=1596 \mathrm{~nm}$.

In a second step, the ridge waveguide has been elaborated using a diamond blade technic in a bulk crystal provided by Cristal Laser SA. The measured transverse section $S$ is found to be non-constant over the $8.6 \mathrm{~mm}$ length along the propagation axis, as shown in Fig. 1 (left).
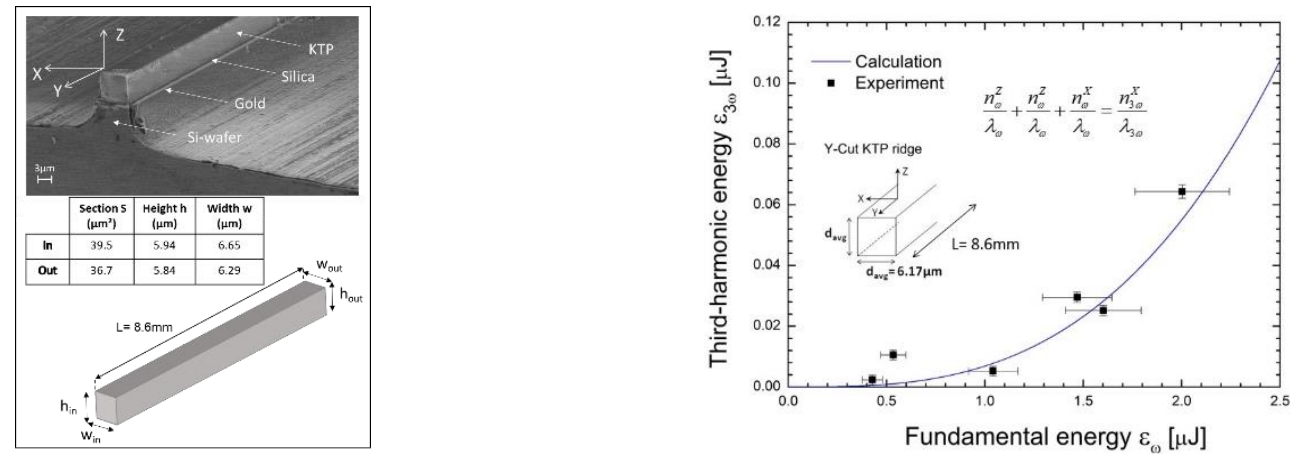

Fig. 1 Left - MEB image, schematic view and dimensions of the KTP ridge waveguide. Right - Measured (black) and calculated (blue) Third-Harmonic energy as a function of the incident fundamental energy.

The third step was devoted to waveguided THG. We used a tunable Optical Parametric Generator as the fundamental beam, with a pulse duration of $15 \mathrm{ps}$ and a repetition rate of $10 \mathrm{~Hz}$. It is injected in the waveguide using a 20 microscope objective (MO). The generated third-harmonic (TH) beam is collected by a $\times 40 \mathrm{MO}$ placed at the exit of the waveguide. The TH energy measured as a function of the fundamental wavelength allowed us to determine that the BPM fundamental wavelength is equal to $1594 \mathrm{~nm}$, which is very close to what was predicted by our model. Figure 1 (right) shows the generated TH energy measured as a function of the fundamental incident energy. The energy conversion efficiency reaches up to $3.4 \%$ for a fundamental energy as low as $20 \mu \mathrm{J}$. The theoretical prediction of the conversion efficiency fully matches the experimental results, and that without any fitting parameters, as shown in Fig. 1 (right). These calculations were performed in the undepleted pump approximation, with evanescent wave losses calculated at $10 \% \mathrm{~cm}^{-1}$ and $30 \% \mathrm{~cm}^{-1}$ for the fundamental and $\mathrm{TH}$ wavelengths respectively, and an effective interaction length of $2.7 \mathrm{~mm}$ measured from the spectral acceptance and allowed us to take into account the gradient of the transverse section along the propagation axis. The THG conversion efficiency can be largely optimized by decreasing the losses, which will be done by increasing the thickness of the silica layer between the gold film and the KTP crystal.

The full agreement between calculation and measurement regarding the THG $(\omega+\omega+\omega \rightarrow 3 \omega)$ BPM conditions and the associated conversion efficiency paves the way for an optimal design of TPG $(3 \omega \rightarrow \omega+\omega+$ $\omega)$ experiments. We expect to generate up to 100 triplets per second at $\lambda_{\omega}^{B P M}=1596 \mathrm{~nm}$ in a 5-cm-long KTP ridge waveguide pumped with $5 \mathrm{~W}$ at $\lambda_{3 \omega}^{B P M}=532 \mathrm{~nm}$ in the $\mathrm{CW}$ regime. A new quantum information frontier is foreseen.

\section{References}

[1] M. Chauvet, F. Henrot, F. Bassignot, F. Devaux, L. Gauthier-Manuel, V. Pecheur, H. Maillotte, and B. Dahmani, High efficiency frequency doubling in fully diced $\mathrm{LiNbO}_{3}$ ridge waveguides on silicon, J. Opt. (United Kingdom), 18, 1 (2016).

[2] V. Boutou, A. Vernay, C. Félix, F. Bassignot, M. Chauvet, D. Lupinski, and B. Boulanger, "Phase-Matched Second-Harmonic Generation in a flux grown KTP crystal ridge optical waveguide,” Opt. Lett., 43(15), 3770 (2018). 